\title{
Hava Tirosh-Samuelson and Christian Wiese (éd.), The Legacy of Hans Jonas. Judaism and the Phenomenon of Life
}

Leiden/Boston, Brill, 2008, 578 pages

\section{Alexander Federau}

\section{OpenEdition}

\section{Journals}

Édition électronique

URL : http://journals.openedition.org/alter/320

DOI : 10.4000/alter.320

ISSN : 2558-7927

Éditeur :

Association ALTER, Archives Husserl (CNRS-UMR 8547)

Édition imprimée

Date de publication : 15 novembre 2014

Pagination : 289-297

ISBN : 978-2-9550449-0-2

ISSN : 1249-8947

\section{Référence électronique}

Alexander Federau, « Hava Tirosh-Samuelson and Christian Wiese (éd.), The Legacy of Hans Jonas. Judaism and the Phenomenon of Life », Alter [En ligne], 22 | 2014, mis en ligne le 01 décembre 2017 consulté le 25 septembre 2020. URL : http://journals.openedition.org/alter/320 ; DOI : https://doi.org/ 10.4000/alter.320

Ce document a été généré automatiquement le 25 septembre 2020.

Revue Alter 


\section{Hava Tirosh-Samuelson and} Christian Wiese (éd.), The Legacy of Hans Jonas. Judaism and the Phenomenon of Life

Leiden/Boston, Brill, 2008, 578 pages

Alexander Federau

\section{RÉFÉRENCE}

Hava Tirosh-Samuelson and Christian Wiese (éd.), The Legacy of Hans Jonas. Judaism and the Phenomenon of Life, Leiden/Boston, Brill, 2008, 578 pages. 
1 La philosophie peut-elle changer le monde? À en écouter Wittgenstein, la réponse est non. La philosophie laisse le monde tel qu'il est. Sa tâche consiste en la résolution des «pseudo-problèmes" qu'elle s'est elle-même posée. Pour Hans Jonas, au contraire, cette manière de philosopher est vaine. Oui, la philosophie peut et doit changer le monde, elle doit même s'emparer des problèmes du moment. Après être devenu un érudit du gnosticisme, Jonas comprend les limites de sa tour d'ivoire et répond à l'appel de son siècle, un $\mathrm{xx}^{\mathrm{e}}$ siècle de bruit et de fureur. Auteur d'une philosophie de la vie qui cherche à renouer le lien avec l'organique, il est surtout célèbre pour son Principe responsabilité qui cherche à bâtir une éthique adaptée à l'âge technologique et qui prend en compte les générations

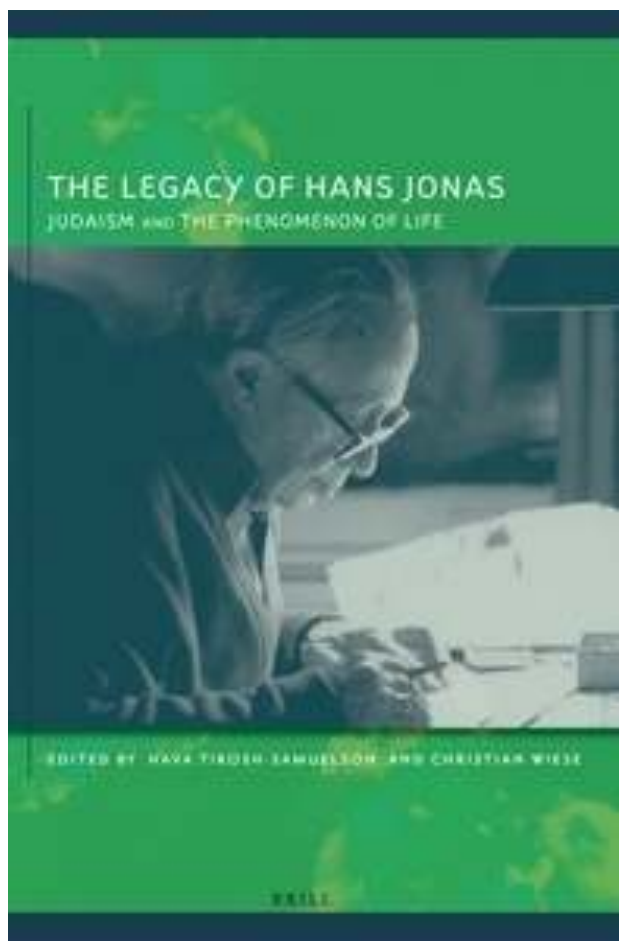
futures.

2 The Legacy of Hans Jonas est le fruit d'un colloque de deux jours tenu les 6 et 7 novembre 2005 à l'Université d'État de l'Arizona et qui a rassemblé aussi bien des historiens, des philosophes, des théologiens, des environnementalistes que des biologistes, dont certains sont des spécialistes de son œuvre. Le format des essais a une originalité : au lieu d'une analyse directe des thèses du philosophe allemand, une approche comparatiste a été privilégiée.

3 Sa pensée est ainsi confrontée avec celle d'un certain nombre de ses contemporains parmi lesquels on compte Buber, Scholem, Anders, Levinas, Watsuji, Leopold ou encore Arne Næss. L'éditrice Hava Tirosh-Samuelson justifie cette démarche par la capacité de Hans Jonas à nouer des amitiés durables, entretenues pendant des décennies et à travers différents continents. Et c'est un fait que le livre fait souvent dialoguer des amis. Un avantage appréciable de cette approche est de laisser percevoir le climat intellectuel d'une époque, au travers des parcours personnels et intellectuels entrelacés. Le choix des contradicteurs est assez stimulant et varié pour qu'on aborde ces dialogues avec une curiosité certaine.

4 Le livre est divisé en trois grandes parties. La première, intitulée «A German-Jewish intellectual ", a un ancrage plus biographique et historique, centré en particulier sur le Marbourg de la République de Weimar. La seconde partie, qui traite tant de biologie théorique, de bioéthique, de philosophie environnementale que de la menace de l'extinction de l'humanité, développe les implications de la philosophie de la vie élaborée par Jonas dans Le phénomène de la vie ; la troisième, plus courte, contient des interventions synthétiques. Le livre contient encore une chronologie, une bibliographie fournie et deux index, des noms propres et des matières, permettant une circulation aisée. Devant l'ampleur des contributions, j'ai choisi de me concentrer ci-après sur des thématiques en liens plus directs avec Le principe responsabilité et les questions environnementales. 


\section{Un philosophe dans la République de Weimar}

La première partie interroge principalement le parcours de Jonas jusqu'en 1933, date où il doit quitter l'Allemagne en raison de la prise de pouvoir nazie. Jonas grandit comme juif allemand dans l'Allemagne de la République de Weimar. Il écrit une thèse philosophique sur le gnosticisme sous la direction de Martin Heidegger. Si comme toute sa génération, il a été d'abord fasciné par le philosophe d'Être et Temps, Jonas prend ensuite ses distances avec son maître. Vittorio Hösle rappelle l'impact de Heidegger sur la thèse de Jonas relative au gnosticisme, puisqu'il y applique les catégories de Dasein, Verfallen ou encore Geworfenheit.

6 Richard Wolin décrit l'éloignement progressif entre Jonas et Heidegger. Jonas finira par rejeter complètement sa philosophie, qu'il qualifiera de nihiliste. Mais pour Hösle, ce rejet n'est qu'apparent. Vouloir surmonter le nihilisme peut encore se comprendre comme une continuation de Heidegger. Hösle argumente à partir de deux thèmes heideggériens de l'œuvre plus tardive de Jonas : une critique de l'ère technique et une analyse de la temporalité. L'originalité de Jonas se situerait alors dans son ontologie de la nature et une éthique pensée comme remède au nihilisme.

7 L'essai de Steven Wasserstrom se focalise sur le groupe de personnes qui composaient les études gnostiques à Marburg et comprenaient des gens comme Martin Plessner, Franz Rosenthal, et Rudolf Bultmann. Wasserstrom montre comment les études gnostiques, un mélange d'orientalisme, d'hellénisme, de théologie, d'histoire et de philosophie, se définissaient en réaction au le néokantisme alors dominant. Wasserstrom considère en particulier Bultmann qui a supervisé la thèse du jeune Jonas. La raison en est qu'en tant que juif, Jonas ne pouvait pas s'inscrire en théologie protestante avec Bultmann, et s'est donc inscrit en philosophie avec Heidegger. C'est d'ailleurs Bultmann qui encourage le jeune Jonas à appliquer les catégories existentielles heideggériennes de manière « scientifique » dans des travaux historiques. Heidegger lui-même était beaucoup plus réticent à cet usage, lui qui professait que « la science ne pense pas».

Cette première partie tient surtout à inscrire Jonas au sein d'une philosophie nationale. Jonas y est présenté comme le dernier des philosophes allemands, puisque le cours de l'histoire fera éclater cette idée d'une philosophie attachée à une nation. La carrière de Jonas aura d'ailleurs été essentiellement internationale, ayant résidé et travaillé dès 1933 dans un cadre anglo-saxon.

\section{Une éthique est-elle possible après Auschwitz?}

Konrad Liessmann entreprend une comparaison avec Günther Anders, surtout connu comme critique de la modernité technique, en particulier dans son combat contre l'industrie nucléaire. Liessmann remarque que Anders et Jonas sont rarement cités ensemble, malgré de nombreuses affinités. Tous deux sont des juifs allemands ayant étudié sous Husserl. Tous deux sont tombés sous le charme de Heidegger avant de le rejeter, et tous deux ont dû fuir l'Allemagne devant la montée du nazisme. Ils ont aimé la même femme, Hannah Arendt, et étaient d'excellents amis. Liessmann reconnaît trois thématiques communes aux deux allemands : une inquiétude quant au destin de 
l'humanité en face de sa puissance technique, une réponse éthique au défi constitué par l'âge technologique. Enfin, une réflexion sur la capacité à croire après la Shoah.

Le premier point se résume en une question : vaut-il la peine de sauver l'humanité ? Oui, selon Jonas, dont l'éthique manifeste l'inquiétude devant sa possible destruction. Par contraste, le pessimisme de Anders est beaucoup plus radical : à ses yeux, il ne peut $\mathrm{y}$ avoir de justification à ce que l'humanité doive continuer à exister. À peine s'il nuance en refusant de l'abandonner totalement à son sort. Le second point concerne une éthique adaptée à un monde technologique. Jonas bâtit son éthique comme un rempart contre une technique qui menace la possibilité d'une vie humaine authentique. Chez Anders, la menace vient plus directement de l'objet technique. Il constate l'invasion d'objets nouveaux dans notre quotidien. Puisque ces objets dictent euxmêmes les usages qu'on va pouvoir en faire et règlent ainsi nos comportements, Anders affirme que l'objet technique en vient à se substituer à l'éthique dans nos vies. La marge de manœuvre se résume désormais à choisir les objets qui nous définiront.

Le dernier point a hanté de nombreux penseurs au sortir de la seconde guerre mondiale. Est-il encore possible de croire en un Dieu après Auschwitz ? Pour Anders, c'est devenu impossible, dans la mesure où, se plaçant du point de vue des victimes, leurs souffrances auraient dû obliger un Dieu à intervenir. Mais puisque cela n'est pas arrivé, il en conclut à l'impossibilité soit de l'existence de Dieu, soit de sa bonté. À partir d'une argumentation similaire, Jonas parvient à une conclusion opposée. Dans "Le concept de Dieu après Auschwitz », il reprend l'idée de l'inintelligibilité d'un Dieu omnipotent qui aurait laissé faire Auschwitz. Mais au lieu de Le nier, il y voit plutôt le signe de Son impuissance, puisqu'il défend l'idée du retrait divin du monde, faisant sien un mythe cabalistique.

\section{Utopie ou responsabilité ?}

12 S'il est un texte que Jonas a critiqué, c'est bien Le principe espérance d'Ernst Bloch. Le succès du Principe responsabilité, dont même le titre évoque la polémique, a jeté une ombre sur l'œuvre d'une vie de Bloch devenue une sorte de repoussoir. Présenter l'utopisme de Bloch de manière charitable comme le fait Michael Löwy ne peut être que louable. Utopiste non repentant, Bloch est convaincu de l'imminence d'un changement révolutionnaire. À partir d'éléments pris au marxisme et au romantisme, Bloch va décrire l'utopie au travers d'un langage qui, pour lui, doit nécessairement être de nature messianique. Löwy remarque que la description précise de l'utopie occupe pourtant peu de place chez Bloch. Sa fonction est avant tout de servir de levier pour critiquer la société industrielle actuelle.

Jonas va critiquer les deux axes de la pensée de Bloch. À propos du romantisme, il pense que l'utopie est dangereuse, en ce qu'elle promet des lendemains qui chantent, alors que les technologies modernes peuvent se révéler menaçantes. À propos du marxisme, dont Jonas se méfie par ailleurs, il reproche surtout à Bloch son anthropocentrisme, reproche assez cocasse quand on sait qu'on le range lui-même souvent à tord parmi les anthropocentristes. Löwy considère que les critiques de Jonas sont parfois justifiées, parfois fragiles ; il est inutile d'opposer utopie et responsabilité : sans principe de responsabilité, l'utopie ne peut être que destructive, mais sans principe d'espoir, la responsabilité n'est rien d'autre qu'une illusion conformiste. 


\section{La responsabilité devant l'altérité : Jonas, Buber, Levinas} concept de responsabilité de Martin Buber, d'Emmanuel Levinas et de Hans Jonas. Si Jonas ne peut pas se ranger parmi ce qu'on a nommé les philosophes du dialogue, les points de contact existent cependant. Tous trois voient les insuffisances de l'éthique kantienne, et tous trois pensent que la dichotomie entre le sujet et l'objet est insuffisante pour aborder l'altérité du point de vue éthique. Enfin, les trois ont une approche phénoménologique. entre le sujet et l'objet caractérise notre perception des objets : c'est le «je-cela ». Mais il existe une seconde modalité, le "je-tu », qui s'établit dans la rencontre avec un autre être. Cette modalité se dévoile dès qu'un dialogue a lieu. Cette relation dialogique ne se résume d'ailleurs pas à un échange de parole. Le dialogue peut aussi être muet, avec un animal, par exemple. Ce qui compte, c'est l'échange avec l'autre.

evinas va reprendre et approfondir ce « je-tu » et le transformer en véritable éthique de l'altérité. L'apparition du visage a toujours une dimension éthique en ce qu'il suscite un appel, la responsabilité devant cet appel étant une composante structurelle de la relation à l'autre, dont le caractère est asymétrique : l'autre apparaît d'une position de hauteur, il a des droits sur moi, son visage signifiant un impératif, qui me rend responsable de l'autre.

Approche analogue chez Jonas qui pense la responsabilité morale qu'il nomme naturelle à partir de l'idée de la valeur intrinsèque des êtres vivants et à partir de l'exemple du nourrisson : voir un nourrisson, c'est percevoir la nécessité d'en prendre soin. L'obligation morale s'impose là aussi de manière asymétrique, puisqu'elle ne peut être réciproque.

La comparaison avec Levinas amène Werner à conclure que ces «intuitionnismes moraux » mènent souvent aux mêmes conclusions. Mais il note aussi des différences. $\mathrm{Si}$ l'apparition d'un «tu» induit des devoirs éthiques, qu'est-ce qui nous dit que ces exigences ne sont pas arbitraires? Comment ce devoir s'harmonise-t-il avec les autres devoirs ? À ses yeux, Levinas ferait appel à un critère théologique en considérant que la dimension du divin se lit dans le visage de l'autre, alors que Jonas, au contraire, refuserait cet expédient théologique au profit d'une philosophie de la vie qui fait une place à une éthique naturalisée.

\section{L'éthique intergénérationnelle}

19 L'essai de William LaFleur traite aussi de la responsabilité, mais dans sa dimension intergénérationnelle. On l'a vu, Jonas fonde son intuition morale sur la relation parentenfant. Selon Richard Wolin, cette prémisse est problématique, car, à ses yeux, elle implique une conception paternaliste du pouvoir politique. La réponse de LaFleur pointe la mécompréhension de Wolin en ce qui concerne la portée à donner à la responsabilité naturelle. Jonas revendique bien une éthique naturelle, qui est une forme de paternalisme, mais il voit bien le danger de la naturalisation de l'éthique, et le principe responsabilité ne se substitue pas à l'idée kantienne selon laquelle une relation morale 
est une relation entre des personnes adultes. La responsabilité naturelle entre en jeu seulement à partir de relations asymétriques, et elle est donc un devoir supplémentaire.

Ce reproche de paternalisme illustre selon LaFleur la difficulté pour la pensée occidentale à faire une place à une éthique intergénérationnelle et il contraste cela avec le Japon. Il rappelle que Jonas a eu une très bonne réception japonaise. Son explication en est que, contrairement à l'Occident, les traditions japonaises intègrent une éthique intergénérationnelle comme le montre sa référence à Watsuji, selon lequel, au Japon, « chaque famille doit avoir un autel bouddhique en honneur des ancêtres [...]. À ces moments-là, même un parent vivant doit se comporter comme un enfant devant le sanctuaire des parents décédés. Ainsi, on ne peut plus dire que le chef de famille est un chef absolu ». Les décisions de celui-ci ne peuvent pas être arbitraires, puisque son devoir est de préserver et de transmettre le patrimoine familial. Watsuji remarque que si cette piété est avant tout rétrospective, elle est en réalité tout autant orientée vers le futur. Le contexte japonais montre comment responsabilité intergénérationnelle n'implique pas nécessairement le recours à une forme de transcendance théologique.

\section{De quelle manière faut-il respecter la nature?}

21 Si le principe responsabilité dépasse le cadre générationnel, s'agit-il pour autant d'une éthique environnementale? Jonas parle la langue des intérêts humains, mais Lawrence Troster va montrer que puisque Jonas reconnaît une valeur intrinsèque à tout être vivant, il s'agit d'une véritable éthique environnementale, du moins si l'on comprend cette dernière comme soucieuse de limiter la puissance humaine en face du monde non-humain. La comparer avec une éthique environnementale, qui a souvent les faveurs des conservationnistes, à savoir l'éthique de la terre d'Aldo Leopold, prend alors tout son sens.

Sans pouvoir la décrire de manière adéquate ici, l'éthique de la terre remarque que tout vivant est pris dans un ensemble de réseaux trophiques au sein d'un écosystème nécessaire à sa survie. Ces réseaux forment selon Leopold une communauté biotique, que son éthique veut préserver. L'idée étant de montrer que l'homme ne se définit pas seulement par son appartenance à des sociétés humaines, mais qu'il fait aussi partie, qu'il est "citoyen", de communautés naturelles plus larges. Chez Jonas, l'enjeu est plutôt de dénoncer la conception moderne de la nature, qui réduit celle-ci à un ensemble de ressources, au profit d'une conception d'intendance qui respecte la valeur de la nature.

Troster va illustrer ces deux interprétations, citoyenne et d'intendance, avec deux psaumes bibliques. Le modèle communautaire de Leopold est relié au psaume 148, qui permet d'établir un lien direct entre communauté et fondement éthique. L'intendance de Jonas est rapportée au psaume 8, qui parle de la bonté de l'univers, comme justification pour la préservation. Les deux psaumes posent la question de la place de l'homme dans le monde. D'un côté, il fait partie d'un écosystème comme membre d'une communauté biotique. De l'autre côté, il a un lien métaphysique au vivant, en tant qu'être vivant lui aussi. 


\section{Du bon usage de Spinoza}

24 Pour changer de sujet, Martin Yaffe considère jusqu'à quel point la philosophie de l'organisme de Jonas a été anticipée par Spinoza. Pour Jonas, le processus du métabolisme qui sert de base à la vie permet d'attribuer une subjectivité non seulement à l'homme, mais aussi à d'autres formes de vie plus primitives. Sa théorie de l'organisme est construite comme une machine de guerre contre le cartésianisme, son dualisme et en particulier son mécanisme - puisque Descartes invitait à penser les animaux comme des machines. Bien que non-spinoziste, Jonas va en revanche travailler à montrer que la doctrine de la substance spinoziste est compatible avec sa philosophie de la vie. Dans le second temps de son analyse, Yaffe va alors comparer cette lecture de Spinoza avec l'interprétation qu'en propose Arne Næss, le théoricien de l'écologie profonde. Selon Yaffe, «si pour Jonas, Spinoza est d'abord d'un intérêt philosophique et ensuite seulement d'un intérêt pour l'éthique environnementale, pour Næss au contraire l'intérêt philosophique est secondaire alors que son éthique environnementale est de prime intérêt ». Selon cette lecture peu charitable, Næss ne s'intéresserait pas au système de Spinoza qu'il jugerait obscur, mais s'en servirait comme «inspiration" pour l'écologie profonde en procédant par choix ciblés de citations compatibles avec le programme de l'écologie profonde.

\section{Conclusion}

Le livre donne des clés importantes pour la compréhension de l'œuvre de Jonas. La mise en contexte historique et intellectuelle est son premier atout. Il s'en dégage la figure d'une pensée résolument allemande, qui se laisse parfois séduire (Heidegger, Kant), parfois tenter (Whitehead, Spinoza), mais dont on voit bien au final l'indépendance. Si Jonas a expliqué lui-même que c'est par rejet du dualisme qu'il est passé des études gnostiques à une philosophie du métabolisme, on comprend ici d'autant mieux comment de cette ontologie du vivant découle à la fois une éthique et une anthropologie.

Bien que cela n'entame en rien de la qualité du livre, le parti pris des éditeurs de mettre l'accent sur la judaïté du philosophe allemand dérange quelque peu. On le remarque dès le sous-titre "Judaïsme et philosophie de la vie ». Les éditeurs se désolent que Jonas ne fasse pas partie du canon des philosophes juifs et un de leurs buts affirmés est "d'entamer un dialogue entre Jonas et les penseurs juifs » (xxviii). Il est entendu que le lien de Jonas au judaïsme est un sujet complexe. Il a été un juif pratiquant et un sioniste militant. Il a traité de questions de théologie juive. Néanmoins, dans la plupart de ses écrits, Jonas parle en tant que philosophe. Il est maintes fois rappelé au sein même de ce recueil comment et pourquoi il refuse l'utilisation de concepts théologiques, au profit d'une approche métaphysique. Et les éditeurs reconnaissent eux-mêmes qu'il a toujours refusé l'appellation de "philosophe juif ». De là, on prend peu de risques à affirmer qu'il aurait voulu qu'on considère son héritage comme legs à la philosophie, et non à la philosophie juive. 